\title{
Minimal Supersymmetric Higgs Bosons with Extra Dimensions as the Source of Reheating and All Matter
}

\author{
Kari Enqvist, ${ }^{1,2}$ Shinta Kasuya, ${ }^{2}$ and Anupam Mazumdar ${ }^{3}$ \\ ${ }^{1}$ Department of Physical Sciences, P.O. Box 64, FIN-00014, University of Helsinki, Finland \\ ${ }^{2}$ Helsinki Institute of Physics, P.O. Box 64, FIN-00014, University of Helsinki, Finland \\ ${ }^{3}$ Physics Department, McGill University, 3600-University Road, Montréal, Canada H3A $2 T 8$ \\ (Received 22 November 2003; published 3 August 2004)
}

\begin{abstract}
We consider the possibility that the dark energy responsible for inflation is deposited into extra dimensions outside of our observable Universe. Reheating and all matter can then be obtained from the minimal supersymmetric standard model flat direction condensate involving the Higgs bosons $H_{u}$ and $H_{d}$, which acquires large amplitude by virtue of quantum fluctuations during inflation. The reheat temperature is $T_{\mathrm{RH}} \lesssim 10^{9} \mathrm{GeV}$ so that there is no gravitino problem. We find a spectral index $n_{s} \approx 1$ with a very weak dependence on the Higgs potential.
\end{abstract}

DOI: 10.1103/PhysRevLett.93.061301

PACS numbers: $98.80 . \mathrm{Cq}, 11.25 . \mathrm{Wx}, 12.60 . \mathrm{Jv}$

The large dark energy of the early inflationary Universe provides us with three things: superluminal stretching of space; quantum fluctuations of scalar fields which may seed density perturbations; and, once the dark energy decays, the origin of all matter. Although, conventionally one relates the spectrum of density perturbations to the properties of the inflaton potential responsible for the early dark energy, recently it has been realized that this is not a necessary condition for a successful inflationary scenario. In curvaton models, the dark energy induces quantum fluctuations in a field whose energy density during inflation is negligibly small but which may later become dominant [1-6]. When the curvaton decays, its isocurvature perturbations will be converted to the usual adiabatic perturbations of the decay products, which thus should ultimately contain also standard model (SM) degrees of freedom. In fact, the logical separation between the field responsible for density perturbations and the geometrical stretching of space was already apparent in pre-big bang models (for a review, see [7]).

The curvaton scenario also points towards the possibility that the inflaton decay products do not necessarily need to give rise to SM particles. This is an issue that relates to the yet unanswered question of "how does the inflaton field couple to SM degrees of freedom?" Indeed, it has been suggested that the inflaton might not couple to the observable sector at all but could within the curvaton framework decay into hidden degrees of freedom $[4,5]$. In such a case, the curvaton field could be found among the flat directions of the minimal supersymmetric standard model (MSSM) (for a review, see [8]). These are described in terms of order parameters, which are combinations of squarks, sleptons, and Higgses, which in the limit of exact supersymmetry (SUSY) have a vanishing potential. The MSSM flat directions have all been classified in [9]. Flatness is lifted by SUSY breaking and by nonrenormalizable terms $[9,10]$, but during inflation the
MSSM flat directions can be effectively massless (this requires the absence of a Hubble-induced mass term $\sim H$ ) and be subject to quantum fluctuations with a spectrum identical to the usual inflaton field. The MSSM curvaton, when it eventually decays, would be a natural explanation for the origin of ordinary matter.

Note that even if the inflaton field did not decay into SM particles, it must decay into something. As a consequence, one would argue that there must be some inflaton-induced reheating with some resulting background energy, thermal or nonthermal, that contributes to the dynamics of the curvaton evolution. Indeed, this is the usual picture addressed in a number of papers [1-6]. However, with the advent of the extra dimensions of brane world and string motivated cosmologies [11], there arises a new possibility: Inflationary dark energy might simply disappear into the bulk.

In the present Letter, we will consider a scenario in which we live on a brane (or a stack of branes) where the stretching of space (of our brane) and the induction of quantum fluctuations of massless fields is sourced by some dynamics in the bulk. We further assume that, once the effective dark energy decays, only an insignificant part of the initial energy density will be deposited on our brane. We will show that the required adiabatic density perturbations can then be obtained from the simplest flat directions involving the MSSM Higgses only. It is obvious that in this case the decay of the curvaton field gives rise to the quarks and leptons, together with cold dark matter in the form of lightest supersymmetric particles.

We do not have a concrete model for brane induced inflation. Many attempts for such a model have been presented in the literature, but all of them have some problems [12-20]. One starting point is the fact that superstring theories admit both stable Bogomol'nyiPrasad-Sommerfeld (BPS) Dp-branes [21] and unstable 
non-BPS branes [22]. Inflation could then be induced by virtue of attraction between a brane-antibrane pair. A pair of brane-antibrane naturally breaks supersymmetry which gives rise to a Coulomb-like interaction if the branes are far apart [13]. Inflation ends when braneantibrane separation becomes of the order of string scale, whence the tachyonic instability ends the inflationary epoch. Reheating due to tachyonic instability primarily reheats the bulk gravitons. The problems and virtues associated with such a setup have recently been discussed in [20], but the process of reheating remains unclear. If the size of the bulk is sufficiently large, then the entire energy of brane-antibrane annihilation or a decay of an unstable brane could be absorbed in the bulk in the form of gravitons [23]. They might still appear to us as a nonthermal background but, if the volume of the bulk is large enough, there could be a suppression effect. If the bulk geometry is warped, the released energy could possibly be deposited on some other, far-away brane.

Inflation may also occur due to a stack of coincident non-BPS Dp-branes [14], which are unstable due to the presence of world volume tachyons. In this picture the tachyon rolls down and reheats the bulk degrees of freedom. Inflation can also occur if the two branes are at an angle, whence the pair of branes could be just Dp. Inflation again ends when there is a tachyon appearing at a critical angle. Reheating in this scenario is quite different [15,17]: Either the branes combine to form a single brane or produce a minimal energy configuration with a conserved charge. The difference in energy between initial and final configuration goes into reheating the bulk degrees of freedom.

A scenario for inflation involving a gas of D-branes embedded in higher dimensions has also been presented [19]. Inflation takes place because the heaviest branes in the spectrum dominate the energy density, which has an equation of state with a negative pressure. Inflation ends in this picture when correlation length set by the branes becomes equal to the Hubble radius. Reheating in this model occurs primarily due to the decay of a brane into the bulk. It seems that, at least within this class of models, it is possible to deposit the effective dark energy as seen by the observer on our brane into the bulk. Another example might be brane world inflation known as steep inflation [24], which has inefficient reheating, or brane embedded in infinite extra dimensions. In the latter case, the emitted gravitons from the brane can be lost forever in the bulk [25]. Yet another interesting scenario could be if the inflaton is charged only under some bulk degrees of freedom, and the bulk is infinitely large, then the energy density of the inflaton decay products can be redshifted away from the brane [26].

Although no fully consistent stingy inflationary and reheating model exists, let us nevertheless assume that after the end of inflation, unlike within the standard picture of scalar inflation, our brane could remain essentially devoid of entropy. While inflation lasts, the effective dark energy triggers field fluctuations along the (MSSM) flat directions. In general, they cannot be excited simultaneously; rather, once a condensate forms in one particular direction, the rest are no longer flat. Hence, a typical situation is where one flat direction is chosen randomly within a single horizon volume which inflates and becomes the observable Universe.

The crucial requirement for an MSSM flat direction to act as a curvaton is that it does not receive a Hubbleinduced mass term during inflation. This is known to hold true at least in D-term inflation [27] and generically in theories with "Heisenberg symmetry" [28]. In the latter case, a one-loop contribution eventually gives a Hubbleinduced mass correction to the flat directions (other than stops) of order $10^{-1} H$ [28], where $H$ is the Hubble rate during inflation. There might be other possibilities also. Let us here just assume that no Hubble mass term is induced during inflation and that the energy scale of the inflaton is $V_{I} \sim H^{2} M_{p}^{2}$, where $M_{p} \sim 2.4 \times 10^{18} \mathrm{GeV}$. After inflation we assume that the energy density goes into exciting the degrees of freedom residing in the bulk.

The flatness of the potential will be lifted by supersymmetry breaking and by nonrenormalizable terms of the form $W=\lambda \Phi^{n} / n M^{n-3}$, where $M$ is a cutoff scale and $n \gtrsim 4$ is the dimensionality of the nonrenormalizable operator; for each flat direction, there exists a set of allowed nonrenormalizable operators (see [8]). In general, the flat direction potential can be written as

$$
V(\phi)=\frac{1}{2} m_{\phi}^{2} \phi^{2}+\frac{\lambda^{2} \phi^{2(n-1)}}{2^{n-1} M^{2(n-3)}},
$$

where $\Phi=\phi e^{i \theta} / \sqrt{2}$ and the first term comes from supersymmetry breaking so that $m_{\phi} \sim \mathrm{TeV}$.

Let us now focus on the simplest MSSM flat direction

$$
H_{u}=\frac{1}{\sqrt{2}}\left(\begin{array}{l}
0 \\
\phi
\end{array}\right), \quad H_{d}=\frac{1}{\sqrt{2}}\left(\begin{array}{l}
\phi \\
0
\end{array}\right) .
$$

We consider only large amplitudes so that we can ignore the $\mu$ term and the Higgs mass terms. Then the effective potential for the $H_{u} H_{d}$ flat direction can be written as

$$
V(\phi)=\lambda^{2} \frac{|\Phi|^{6}}{M_{p}^{2}},
$$

where we parametrize the expectation value of the flat direction as $\Phi\left(=\phi e^{i \theta} / \sqrt{2}\right)$. Here $\lambda$ is a constant of $O(1)$ which we set to unity for simplicity.

During inflation, when the cosmological scales leave the horizon, the curvature of the potential should be small enough for the fluctuation not to be damped [6]. The amplitude of the fluctuation should also be of the correct order of magnitude. Hence, we require that 


$$
V^{\prime \prime}\left(\phi_{*}\right)=\beta^{2} H_{*}^{2}, \quad \frac{H_{*}}{\phi_{*}}=\delta,
$$

where $H$ is the Hubble parameter, and the prime denotes the derivative with respect to the field $\phi$. The star denotes the value evaluated at the horizon crossing, and $\beta \ll 1$ and the perturbation $\delta \sim 10^{-5}$. Thus, we obtain

$$
\phi_{*} \sim \beta \delta M_{p}, \quad H_{*} \sim \beta \delta^{2} M_{p} .
$$

Therefore we find the scale of inflationary dark energy to be $V_{I}^{1 / 4} \sim\left(H_{*} M_{p}\right)^{1 / 2} \sim \beta^{1 / 2} \delta M_{p}$.

According to our assumption, after inflation there is no energy density on our brane except for the condensate along the $H_{u} H_{d}$ flat direction. Once inflation is over, the condensate starts to oscillate and eventually decays. The maximum temperature which can be achieved after the decay is $T_{\max } \sim\left[V\left(\phi_{*}\right)\right]^{1 / 4}$. Notice that it is much smaller than the scale of inflation. $T_{\max }$ corresponds to an instantaneous decay of the flat direction into a thermal bath of MSSM particles. We will show below that this indeed is the case.

The Higgs flat direction couples, respectively, to MSSM fermions and bosons as $f \phi \psi \psi$ and $f^{2} \phi^{2} \chi^{2}$, where $f$ represents gauge or Yukawa coupling constant. After inflation, the amplitude of the flat direction is very large so one expects both fermionic and bosonic preheating [29]. Preheating in the context of MSSM flat direction has been discussed in [30]. The $q$ parameter in this case is given by

$$
q \sim \frac{f^{2} \phi_{*}^{2}}{\omega^{2}} \sim f^{2}\left(\frac{M_{p}}{\phi_{*}}\right)^{2} \gg 1,
$$

where $\omega=\sqrt{V^{\prime \prime}\left(\phi_{*}\right)}$. Typically, the momentum which the produced particle carries is $k_{\text {res }} \sim \omega q^{1 / 4}$. Since the occupation numbers of fermion and boson are $n_{k}^{(f)} \sim 1$ and $n_{k}^{(b)} \sim 1 / f^{2}$, respectively, the energy density may be estimated as

$$
\begin{aligned}
& \rho_{f} \sim n_{k}^{(f)} \omega^{4} q \sim f^{2} \omega^{2} \phi^{2} \sim f^{2} \rho_{\phi}, \\
& \rho_{b} \sim n_{k}^{(b)} \omega^{4} q \sim \omega^{2} \phi^{2} \sim \rho_{\phi} .
\end{aligned}
$$

Back reaction terminates the resonant particle production when the energy density of the produced particle becomes of the order of the energy density of the oscillating condensate. Notice that there is less decrease of the energy density due to cosmic expansion during preheating in this case, because the Hubble parameter is much smaller than the curvature of the potential for the flat direction: $H \sim \omega\left(\phi_{*} / M_{p}\right) \ll \omega$.

Once MSSM fermions and bosons are produced, the energy density of (nonthermal) radiation evolves as $\rho_{\text {rad }}=\rho_{f}+\rho_{b} \propto a^{-4}$, while the residual oscillation of the flat direction decreases as $\rho_{\phi} \propto a^{-9 / 2}$ [31], where $a(t)$ is the scale factor of the Universe. After the mass term in the effective potential of the flat direction dominates, the latter changes its evolution to $\rho_{\phi} \propto a^{-3}$. The energy densities of radiation and the residual flat direction condensate equalize when $\phi=\phi_{\mathrm{eq}} \sim(\beta \delta)^{-1} m_{\phi}$ and, if the decay of the flat direction takes place before this time, the contribution to the total radiation density is subdominant. This happens if

$$
f \gtrsim \sqrt{8 \pi}\left(\frac{\phi_{\mathrm{eq}}}{M_{p}}\right)^{1 / 2} \sim \sqrt{8 \pi}(\beta \delta)^{-(1 / 2)}\left(\frac{m_{\phi}}{M_{p}}\right)^{1 / 2} .
$$

For $\beta=0.1$ and $\delta=10^{-5}$, the right-hand side reads $\sim 10^{-4}$. Since the Higgs has couplings much larger than $10^{-4}$ (e.g., gauge couplings), the $H_{u} H_{d}$ flat direction decays well before the equality time. Hence, the amount of radiation is totally determined by the preheating era.

Let us now estimate the reheat temperature. Since the maximum temperature is $T_{\max } \sim\left[V\left(\phi_{*}\right)\right]^{1 / 4}$, we may write

$$
T_{R H} \lesssim T_{\max } \sim 10^{9}\left(\frac{\beta}{0.1}\right)^{3 / 2}\left(\frac{\delta}{10^{-5}}\right)^{3 / 2} \mathrm{GeV},
$$

although, depending on the thermalization process, the true reheat temperature could be much lower than $10^{9} \mathrm{GeV}$. For a generic $n=6(n=7)$ direction, the maximum reheat temperature would be $10^{13}\left(10^{14}\right) \mathrm{GeV}$, so that the avoidance of the gravitino problem [32] is not automatic in general. However, in the particular case of the $H_{u} H_{d}$ direction there is no gravitino problem.

Regarding the density perturbations, the amplitude of the fluctuation created during inflation will be imprinted on radiation. The spectral index of microwave temperature perturbations can then be evaluated as [33]

$$
n_{s}-1=2 \frac{\dot{H}_{*}}{H_{*}^{2}}+\frac{2}{3} \frac{V^{\prime \prime}\left(\phi_{*}\right)}{H_{*}^{2}} .
$$

In our setup, the change of the Hubble parameter is negligible. Hence, we find $n_{s}-1 \approx 0.007$ for $\beta=0.1$, which gives a very flat spectrum consistent with the recent Wilkinson microwave anisotropy probe observation of $n_{s}=0.99 \pm 0.04$ [34].

One should note that the $H_{u} H_{d}$ direction has a vanishing baryon and lepton number. Hence, there is no baryonic nor leptonic isocurvature problem [35]. There is no Affleck-Dine baryogenesis either but, since the reheat temperature $\gg \mathcal{O}(1) \mathrm{TeV}$, baryons could be produced by sphaleron processes at the electroweak phase transition.

In conclusion, we have pointed out that in theories with extra dimensions there may exist the possibility that the effective dark energy which drives inflation could be deposited outside of our observable brane. As a consequence, reheating and all matter could originate simply from a MSSM flat direction involving the Higgses, which gets excited during inflation. Moreover, as we showed, the reheat temperature is low enough to avoid the gravitino problem. 
It is interesting to note that the spectral index Eq. (10) depends - albeit very weakly — on the Higgs potential, which may not be observable soon but nevertheless demonstrates that, in principle, MSSM curvaton models could be tested by laboratory experiments. Such connections between the properties of elementary particles and temperature fluctuations of the microwave sky would naturally be highly desirable. It remains to be seen whether inflationary models based on string theory can allow for the depositing of the dark energy outside of our brane as required in the present framework.

K. E. is supported partly by the Academy of Finland Grant No. 75065, and A. M. acknowledges Abdel Lorenzana for helpful discussion.

[1] K. Enqvist and M. S. Sloth, Nucl. Phys. B626, 395 (2002).

[2] D. H. Lyth and D. Wands, Phys. Lett. B 524, 5 (2002).

[3] T. Moroi and T. Takahashi, Phys. Lett. B 522, 215 (2001); 539, 303(E) (2002)]; D. H. Lyth, C. Ungarelli, and D. Wands, Phys. Rev. D 67, 023503 (2003); N. Bartolo and A. R. Liddle, Phys. Rev. D 65, 121301 (2003); A. Hebecker, J. March-Russell, and T. Yanagida, Phys. Lett. B 552, 229 (2003); R. Hofmann, hep-ph/0208267; K. Dimopoulos and D. H. Lyth, hep-ph/0209180; T. Moroi and H. Murayama, Phys. Lett. B 553, 126 (2003); M. Bastero-Gil, V. Di Clemente, and S. F. King, Phys. Rev. D 67, 103516 (2003); K. A. Malik, D. Wands, and C. Ungarelli, Phys. Rev. D 67, 063516 (2003); M. Postma, Phys. Rev. D 67, 063518 (2003); C. Gordon and A. Lewis, Phys. Rev. D 67, 123513 (2003); B. Feng and M. z. Li, Phys. Lett. B 564, 169 (2003); K. Dimopoulos, D. H. Lyth, A. Notari, and A. Riotto, J. High Energy Phys. 07 (2003) 053; A. R. Liddle and L. A. Urena-Lopez, Phys. Rev. D 68, 043517 (2003); J. McDonald, Phys. Rev. D 68, 043503 (2003); K. Dimopoulos, Phys. Rev. D 68, 123506 (2003); K. Dimopoulos, G. Lazarides, D. Lyth, and R. Ruiz de Austri, J. High Energy Phys. 05 (2003) 057; M. Endo, M. Kawasaki, and T. Moroi, Phys. Lett. B 569, 73 (2003); S. Kasuya, M. Kawasaki, and F. Takahashi, Phys. Lett. B 578, 259 (2004); K. Hamaguchi, M. Kawasaki, T. Moroi, and F. Takahashi, hep-ph/0308174; D. H. Lyth, Phys. Lett. B 579, 239 (2004); J. McDonald, hep-ph/0310126; A. Mazumdar, hep-th/0310162; M. Axenides and K. Dimopoulos, hep-ph/0310194; M. Giovannini, hep$\mathrm{ph} / 0310024$.

[4] K. Enqvist, S. Kasuya, and A. Mazumdar, Phys. Rev. Lett. 90, 091302 (2003).

[5] K. Enqvist, A. Jokinen, S. Kasuya, and A. Mazumdar, Phys. Rev. D 68, 103507 (2003).

[6] K. Dimopoulos, G. Lazarides, D. H. Lyth, and R. Ruiz de Austri, Phys. Rev. D 68, 123515 (2003).
[7] J. E. Lidsey, D. Wands, and E. J. Copeland, Phys. Rep. 337, 343 (2000); M. Gasperini and G. Veneziano, Phys. Rep. 373, 1 (2003).

[8] K. Enqvist and A. Mazumdar, Phys. Rep. 380, 99 (2003).

[9] T. Gherghetta, C. Kolda, and S. P. Martin, Nucl. Phys. B468, 37 (1996).

[10] M. Dine, L. Randall, and S. Thomas, Nucl. Phys. B458, 291 (1996).

[11] F. Quevedo, Classical Quantum Gravity 19, 5721 (2002).

[12] G. R. Dvali and S. H. Tye, Phys. Lett. B 450, 72 (1999).

[13] C. P. Burgess, M. Majumdar, D. Nolte, F. Quevedo, G. Rajesh, and R. J. Zhang, J. High Energy Phys. 07 (2001) 047.

[14] A. Mazumdar, S. Panda, and A. Perez-Lorenzana, Nucl. Phys. B614, 101 (2001).

[15] J. Garcia-Bellido, R. Rabadan, and F. Zamora, J. High Energy Phys. 01 (2002) 036.

[16] G. Shiu and S. H. Tye, Phys. Lett. B 516, 421 (2001).

[17] N. Jones, H. Stoica, and S. H. Tye, J. High Energy Phys. 07 (2002) 051.

[18] K. Dasgupta, C. Herdeiro, S. Hirano, and R. Kallosh, Phys. Rev. D 65, 126002 (2002).

[19] R. Brandenberger, D. A. Easson, and A. Mazumdar, hepth/0307043.

[20] S. Kachru, R. Kallosh, A. Linde, J. Maldacena, L. McAllister, and S. P. Trivedi, J. Cosmol. Astropart. Phys. 10 (2003) 013.

[21] J. Polchinski, String Theory (Cambridge University Press, Cambridge, England, 1998).

[22] A. Sen, J. High Energy Phys. 12 (1999) 027.

[23] N. Lambert, H. Liu, and J. Maldacena, hep-th/0303139.

[24] E. J. Copeland, A. R. Liddle, and J. E. Lidsey, Phys. Rev. D 64, 023509 (2001).

[25] G. R. Dvali and G. Gabadadze, Phys. Rev. D 63, 065007 (2001).

[26] A. Mazumdar and A. Perez-Lorenzana, hep-ph/0311106.

[27] P. Binetruy and G. Dvali, Phys. Lett. B 388, 241 (1996).

[28] M. K. Gaillard, H. Murayama, and K. A. Olive, Phys. Lett. B 355, 71 (1995).

[29] L. Kofman, A. D. Linde, and A. A. Starobinsky, Phys. Rev. D 56, 3258 (1997); P. B. Greene and L. Kofman, Phys. Rev. D 62, 123516 (2000).

[30] M. Postma and A. Mazumdar, J. Cosmol. Astropart. Phys. 01 (2004) 005.

[31] M. Turner, Phys. Rev. D 28, 1243 (2000).

[32] J. Ellis, J. E. Kim, and D.V. Nanopoulos, Phys. Lett. B 145, 181 (1984); A. L. Maroto and A. Mazumdar, Phys. Rev. Lett. 84, 1655 (2000); R. Kallosh et al., Phys. Rev. D 61, 103503 (2000).

[33] D. H. Lyth and D. Wands, Phys. Lett. B 524, 5 (2002).

[34] D. N. Spergel et al., Astrophys. J. Suppl. Ser. 148, 175 (2003).

[35] K. Enqvist and J. McDonald, Phys. Rev. Lett. 83, 2510 (1999); K. Enqvist and J. McDonald, Phys. Rev. D 62, 043502 (2000); M. Kawasaki and F. Takahashi, Phys. Lett. B 516, 388 (2001). 